\title{
The Sclerotinia sclerotiorum FoxE2 Gene Is Required for Apothecial Development
}

\author{
Lu Wang, Yanzhi Liu, Jinliang Liu, Yanhua Zhang, Xianghui Zhang, and Hongyu Pan
}

College of Plant Sciences, Jilin University, Changchun, 130062.

Accepted for publication 3 January 2016.

\begin{abstract}
Wang, L., Liu, Y., Liu, J., Zhang, Y., Zhang, X., and Pan, H. 2016. The Sclerotinia sclerotiorum FoxE2 gene is required for apothecial development. Phytopathology 106:484-490.

Sclerotinia sclerotiorum is a widely dispersed plant pathogenic fungus causing many diseases such as white mold, Sclerotinia stem rot, stalk rot, and Sclerotinia head rot on many varieties of broadleaf crops worldwide. Previous studies have shown that the Forkhead-box transcription factors (FOX TFs) play key regulatory roles in the sexual reproduction of some fungi. Ss-FoxE2 is one of four FOX TF family member genes in $S$. sclerotiorum. Based on ortholog function in other fungi it is hypothesized to function in $S$. sclerotiorum sexual reproduction. In this study, the role of Ss-FoxE2 in S. sclerotiorum was identified with a gene

knock-out strategy. Following transformation and screening, strains having undergone homologous recombination in which the hygromycin resistance gene replaced the gene Ss-FoxE2 from the genomic DNA were identified. No difference in hyphae growth, number, and weight of sclerotia and no obvious change in virulence was observed among the wild type Ss-FoxE2 knock-out mutant and genetically complemented mutant; however, following induction of sclerotia for sexual development, apothecia were not formed in Ss-FoxE2 knock-out mutant. The Ss-FoxE2 gene expressed significantly higher in the apothecial stages than in other developmental stages. These results indicate that Ss-FoxE2 appears to be necessary for the regulation of sexual reproduction, but may not affect the pathogenicity and vegetative development of S. sclerotiorum significantly.
\end{abstract}

Sclerotinia sclerotiorum (Lib.) de Bary is a cosmopolitan necrotrophic fungal pathogen attacking many varieties of plants and causing diseases on 408 plant species from 278 genera and 75 families (Boland and Hall 1994). Because of the extensive crop loss, broad host range, and the difficulty of controlling diseases caused by this fungus, sustained research efforts have focused on this pathogen (Kabbage et al. 2015; Xiao et al. 2014). The formation of pigmented, multihyphal sclerotia is required for its long-term survival. The sclerotium is an aggregate of filamentous hyphae, which are hardened by a thin rind layer and contain nutrient reserve materials. Sclerotia also serve as a supporting tissue for germination, are able to survive in the soil for many years (Adams and Ayers 1979; Chet and Henis 1975; Willetts and Bullock 1992; Willetts and Wong 1980). Millions of airborne ascospores, produced by and released from the apothecium (the sexual fruiting body) are the major supply of inoculum in many Sclerotinia diseases and are critical for spreading the disease in the field. Therefore, blocking sclerotia or apothecium development might be an effective method to control Sclerotinia diseases.

Research has been concentrated on identifying the physiological factors that can affect sclerotial development (Chet and Henis 1975; Willetts and Bullock 1992; Willetts and Wong 1980; Xiao et al. 2014) because of the importance of sclerotia in the disease cycle of $S$. sclerotiorum. Other studies have been designed to determine the potential molecular and biochemical mechanisms related to the regulation of sclerotial morphogenesis (Chen and Dickman 2005; Chen et al. 2004; Georgiou et al. 2006; Li and Rollins 2010; Patsoukis

Corresponding authors: X. Zhang and H. Pan;

E-mail addresses: zhangxianghui@jlu.edu.cn and panhongyu@jlu.edu.cn

First and second authors contributed equally to this work.

*The $\boldsymbol{e}$-Xtra logo stands for "electronic extra" and indicates that one supplementary figure and one supplementary table are published online.

http://dx.doi.org/10.1094/PHYTO-08-15-0181-R

(C) 2016 The American Phytopathological Society and Georgiou 2007; Rollins 2003). In particular, phosphorylative relay involving cAMP-dependent processes, ERK-like mitogen-activated protein kinase, and Ser/Thr phosphatases type 2A and 2B (Erental et al. 2007; Harel et al. 2006; Jurick et al. 2004) play key roles in regulation of sclerotia development. Xiao et al. (2014) shows results that suggest that $S$ s-cafl plays a pivotal role in the formation of compound appressorium and sclerotia development in S. sclerotiorum (Xiao et al. 2014). In S. sclerotiorum the sclerotium plays a key role in transitioning from asexual to sexual development. Apothecia are produced on the surface of mature sclerotia but few critical genes or signal transduction pathways have been reported for apothecia germination.

Forkhead box (FOX) is a large transcription factor (TF) family with key regulatory roles in animals and fungal (Lam et al. 2013). FOX genes encode TFs containing a forkhead helix-turn-helix DNA binding domain of $\sim 110$ amino acids and are involved in many cellular processes, such as morphogenesis and cell cycle regulation (Wang et al. 2015). From previous studies, FOX TFs were found in animal and fungal genomes, but not in plants (Shimeld et al. 2010). Koranda et al. (2000) characterized four FOX TFs in Saccharomyces cerevisiae: FKH1, FKH2, HCM1, and FHL1. They found that FKH1 and FKH 2 function in $\mathrm{G} 2$ and $\mathrm{M}$ phases of cell cycle, and the fkh1 and fkh2 mutants display abnormal morphology (Koranda et al. 2000; Kumar et al. 2000; Zhu et al. 2000). On the other hand, forkhead protein $\mathrm{Hcm} 1$ was reported to be involved in chromosome segregation (Pramila et al. 2006). Fkh2, a forkhead TF, was also reported to have a regulatory function in the $\mathrm{M}$ and $\mathrm{G}(1)$ phases of cell division in Schizosaccharomyces pombe, which was also important for cell morphology and separation (Bulmer et al. 2004). Three FOX TFs $(f k h F, f k h E$, and $f h p A)$ have been reported to play key roles in asexual development in Aspergillus nidulans (Lee et al. 2005; Park et al. 2010). But homologs of these genes are not present in the S. sclerotiorum genome.

On the basis of these observations, we hypothesize that FOX TFs in S. sclerotiorum play a key role in regulating sexual development. In order to illuminate the functions of FOX TFs in S. sclerotiorum, 
we conducted sequence analysis and functional characterization of the FoxE2 gene in S. sclerotiorum. The goal of our study was to test this hypothesis by deleting FoxE2 allele in S. sclerotiorum and characterizing phenotypes of the mutants.

\section{MATERIALS AND METHODS}

Strains and media. All strains used in this study were derived from strain JRUF1 of S. sclerotiorum provided by J. Rollins, University of Florida. This isolate readily forms apothecia and was used as the wild type. Potato dextrose agar (PDA) was used to routinely grow cultures at $25^{\circ} \mathrm{C}$. PDA medium supplemented with hygromycin B at $100 \mu \mathrm{g} / \mathrm{ml}$ (Roche, China) was used to culture transformation mutants. Hyphae stocks were maintained as desiccated mycelia-colonized filter paper at $-20^{\circ} \mathrm{C}$ and as dry sclerotia at $4^{\circ} \mathrm{C}$. PDA culture-derived sclerotia was used to induce apothecia using the method previously described ( $\mathrm{Li}$ and Rollins 2010).

Isolation and manipulation of nucleic acids. A modified CTAB protocol was used to extract fungal genomic DNA (Proctor et al. 1997). All plasmid DNAs were propagated using Escherichia coli $\mathrm{DH} 5 \alpha$. Standard protocols were followed to conduct plasmid isolations, agarose gel electrophoresis, DNA restriction digests, ligation reactions, E. coli transformations, and Southern blot analyses (Sambrook and Russell 2001). All gel fragments were purified with gel extraction kits (Tiangen, Beijing). Trizol reagents (Dingguo, Beijing) were used to extract total RNA from lyophilized mycelia, sclerotia, and apothecia tissues according to the manufacturer's instructions. DNA sequences were determined by Boshi Biotechnology Company (Harbin, China).

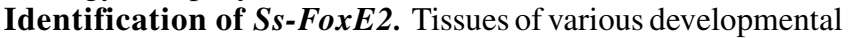
stages of $S$. sclerotiorum including hyphae, sclerotia, and apothecia were harvested for total RNA preparations using Trizol reagent (Dingguo, Beijing). The fungal cDNAs were synthesized using M-MLV reverse transcription (TaKaRa, Dalian) with oligo-d $\left(\mathrm{T}_{18}\right)$ primers. Ss-FoxE2 cDNA (XM_001592862.1, XP_001592912.1, and SS1G_05834) without a stop codon was amplified with Ex Taq DNA polymerase (TaKaRa, Dalian) with the coding sequencespecific primers Ssfoxe2-eGFP-F and Ssfoxe2-eGFP-R (Table 1). The primers contained $S a c I$ and $S m a I$ restriction enzyme sites, respectively, in the $5^{\prime}$ ends for vector construction. The PCR product was purified and ligated into pMD18-T cloning vector (TaKaRa, Dalian). The recombinant plasmids were sequenced by Boshi Biotechnology Company (Harbin, China).

Gene replacement. For functional analysis of $S s-F o x E 2$, we targeted the gene for knock-out by double homologous recombination. Table 1 shows the gene-specific primers used to amplify the 0.9-kb 5'-UTR of the Ss-FoxE2 gene with added KpnI and EcoRI restriction enzyme sites (F2-L-F and F2-L-R, underlined in Table 1) and 1.0-kb 3'-UTR Ss-FoxE2 gene with added BamHI and XbaI restriction enzyme sites (F2-R-F and F2-R-R, underlined in Table 1) from the $S$. sclerotiorum genomic DNA. These two fragments were cloned into vector $\mathrm{pXEH}$ with hygromycin phosphotransferase (hph) cassette containing trpC promoter and named as pXEH-R-L. Protoplasts of S. sclerotiorum were prepared and transformed as previously described (Rollins 2003). Hygromycin-resistant colonies were screened by PCR with primers H1, H2, F2-F, F2-R, Hpt-a, Hpt-b, F2-L-F, and F2-R-R (Table 1) to identify independent strains disrupted in Ss-FoxE2. All transformants were hyphal-tip transferred at least three times.

Construction of complementation strains. For genetic complementation of the Ss-FoxE2 knockout mutant, primers C-F2-F and C-F2-R were used to amplify a 3.4-kb fragment with NotI and NheI restriction enzyme sites (underlined in Table 1) containing the full length Ss-FoxE2 open reading frame flanked with 5 ' - and $3^{\prime}$-UTR sequences (Table 1). This 3.4-kb fragment was cloned into vector pD-NEO1 (modified from vector pD-NAT1 and pSilent Dual1) for transformation with geneticin at $100 \mu \mathrm{g} / \mathrm{ml}$. The restriction enzyme digestions, gel electrophoresis, DNA fragment purifications, and ligations were performed using standard procedures (Sambrook and Russell 2001).

Southern blot analysis. In Southern blot analysis, genomic DNA was probed with the hygromycin resistance cassette from pXEH and the partial sequence of the open reading frame of gene Ss-FoxE2. The first probe of a 712-bp amplicon was designed from the open reading frame of the $S s-F o x E 2$ gene using primers F2-F and

\section{A Probe 1}
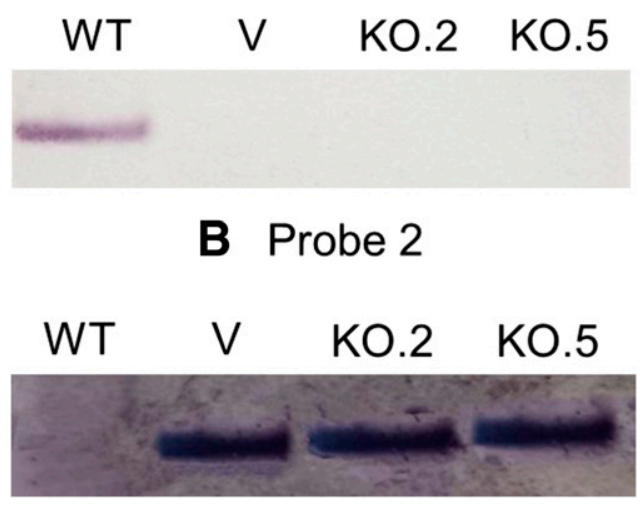

Fig. 1. Southern hybridization analysis of Ss-FoxE2 knock-out mutants of Sclerotinia sclerotiorum with probe 1 and probe 2. Probe 1: Ss-FoxE2 open reading frame. Probe 2: hygromycin-resistance cassette. WT: wild-type JRUF-1. V: knock-out vector plasmid. KO.2 and KO.5: Ss-FoxE2 knock-out mutants strains.

TABLE 1 . The sequence of primers in this study

\begin{tabular}{|c|c|c|}
\hline Primer name & Primer sequence & Annotation \\
\hline F2-L-F & GGGTACCTAAGGAGCAGTGGGAACG (KpnI) & 5'-UTR forward primer of Ss-FoxE2 \\
\hline F2-L-R & GEAATTCTGCAGAAGTCATGGAGGTG (EcoRI) & $5^{\prime}$-UTR reverse primer of $S s$-FoxE2 \\
\hline F2-R-F & CTCTAGATTGACATCTGTTCGCAGCAC (XbaI) & 3'-UTR forward primer of Ss-FoxE2 \\
\hline F2-F & CTTTGATGACGGGACTGT & Forward primer of partial Ss-FoxE2 \\
\hline F2-R & ATGCTCCGAGGAAGATGG & Reverse primer of partial Ss-FoxE2 \\
\hline H1 & CGTCTGCTGCTCCATACAAG & Forward primer of $h p h$ \\
\hline $\mathrm{H} 2$ & GCGAAGAATCTCGTGCTTTC & Reverse primer of $h p h$ \\
\hline C-F2-F & GCTAGCAGTCATGGGGTGGGAGA (NheI) & Forward primer of $S s-F o x E 2$ complementation \\
\hline C-F2-R & GCGGCCGCTGGAGCTGAAGCACGATA (NotI) & Reverse primer of $S s-F o x E 2$ complementation \\
\hline Ssfoxe2-eGFP-F & CGAGCTCATGGATCCGATCACTTTCAG (SacI) & Forward primer of eGFP for $S s$-FoxE2 \\
\hline Ssfoxe2-eGFP-R & TCCCCCGGGAGTATTGGTTATTGGTGC (SmaI) & Reverse primer of eGFP for $S s-F o x E 2$ \\
\hline qSs-FoxE2-F & GGATTCCGATCACTTTCAGCAG & Forward primer of RT-PCR for Ss-FoxE2 \\
\hline qSs-FoxE2-R & GCAGTCATTGTTGGGCATAGG & Reverse primer of RT-PCR for $S s-F o x E 2$ \\
\hline qSs-Histone-F & GGCTCGTACCAAGCAAACTG & Forward primer of histone for $S s-F o x E 2$ \\
\hline
\end{tabular}


F2-R (Table 1), and the second probe of a 646-bp amplicon was designed from the $h p h$ gene using primers $\mathrm{H} 1$ and $\mathrm{H} 2$ (Table 1).

Expression level of the $S$ s-FoxE2 in S. sclerotiorum life cycle. A previous study showed that sclerotium development was divided into six sequential stages ( $\mathrm{S} 1$ to $\mathrm{S} 6$ ) and seven sequential stages (A1 to A7) for apothecium development (Li and Rollins 2009). In our study, we reassigned the stages $S 1$ to $S 2, S 3$ to S4, and S5 to S6 classified by Li and Rollins (2009) as stages SI, SII, and SIII, respectively. Similarly, we reassigned apothecium stages A1 to A3, A4 to A5, and A6 to A7 as stages AI, AII, and AIII, respectively. To evaluate the expression levels of Ss-FoxE2 in sequential stages in the life cycle of $S$. sclerotiorum by real-time PCR, total RNA from tissues collected were extracted using Trizol reagent (Dingguo Beijing). One microgram of total RNA was prepared for reverse

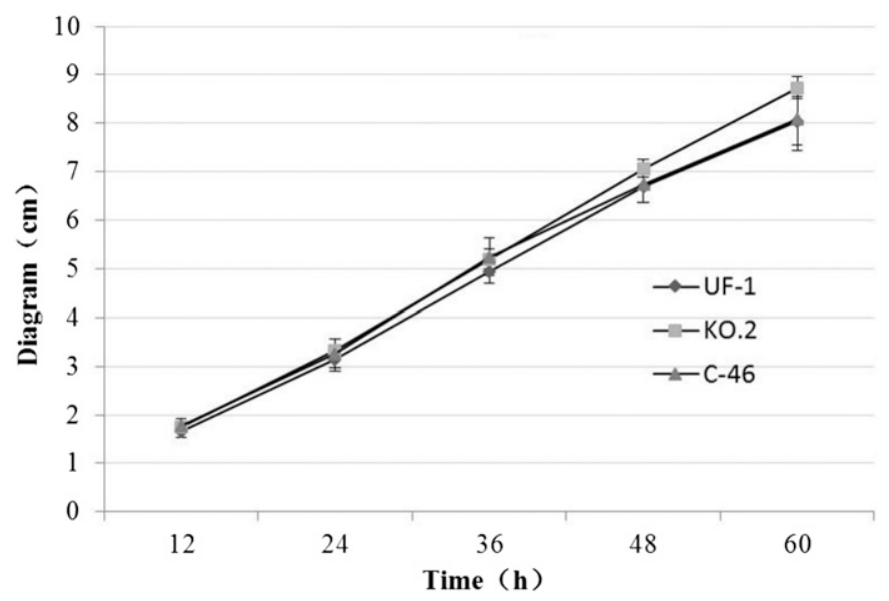

Fig. 2. Growth rate of wild-type, $S s-F o x E 2$ knock-out mutant KO.2 and Ss-FoxE2 complemented mutant C-46. transcription using PrimeScript RT reagent Kit with cDNA Eraser (Perfect Real Time; TaKaRa). Relative quantification of gene expression was performed with the SYBR Green Real-Time PCR using SYBR Green Reagent kit (TaKaRa) with the Applied Biosystems 7500 Real-Time PCR detection system according to the manufacturer's protocol. Primers used for Ss-FoxE2 gene were Ss-FoxE2 (qSsFoxE2-F and qSsFoxE2-R) and housekeeping gene Ss-HistoneH3 (XM_001589836.1 and SS1G_09608) were qSsHistone-F and qSsHistone-R (Table 1). These primers were designed with Primer Premier 5.0 software (Premier Biosoft International, Palo Alto, CA) to produce an amplification product of $196 \mathrm{bp}$ for Ss-FoxE2 and $235 \mathrm{bp}$ for Ss-HistoneH3. Each real-time PCR mixture contained $10 \mu \mathrm{l}$ of SYBR Premix Ex Taq TM II (2×), $2 \mu \mathrm{l}$ of 20-fold diluted cDNA, 4-pmol concentration of each primer, $0.4 \mu \mathrm{l}$ of ROX Reference Dye II (50x), and nuclease-free water, to a final volume of $20 \mu \mathrm{l}$. Total cDNA abundance in the samples was normalized using the Ss-HistoneH3 gene as an internal reference. Amplification conditions were as follows: $30 \mathrm{~s}$ at $95^{\circ} \mathrm{C}$, then 40 cycles consisting of $5 \mathrm{~s}$ at $95^{\circ} \mathrm{C}, 40 \mathrm{~s}$ at $60^{\circ} \mathrm{C}$, and $15 \mathrm{~s}$ at $95^{\circ} \mathrm{C}$, and then $1 \mathrm{~min}$ at $60^{\circ} \mathrm{C}$ and $15 \mathrm{~s}$ at $95^{\circ} \mathrm{C}$. The $2^{-\Delta \Delta \mathrm{CT}}$ method was used to analyze the relative quantification of gene expression (Schmittgen et al. 2000). Each sample was repeated three times. The real-time PCR experiment was replicated at least three times.

Hyphae and sclerotium morphology. The wild type, Ss-FoxE2 knock-out, and complementation strains were cultured on PDA plates and used for phenotype analysis. Colony diameters were measured over time to determine the radial mycelial growth on PDA plates, which were inoculated in the center with an agar-mycelium plug derived from the growing margin of PDA culture of each strain. Hyphal morphology and infection cushion were analyzed by optical microscope. Seven to ten days after inoculation, sclerotia were collected, counted, and dried, and then the dry weight of sclerotia was measured.

Apothecial germination assay. Mature sclerotia of each strain (the wild type, Ss-FoxE2 knock-out, and complementation

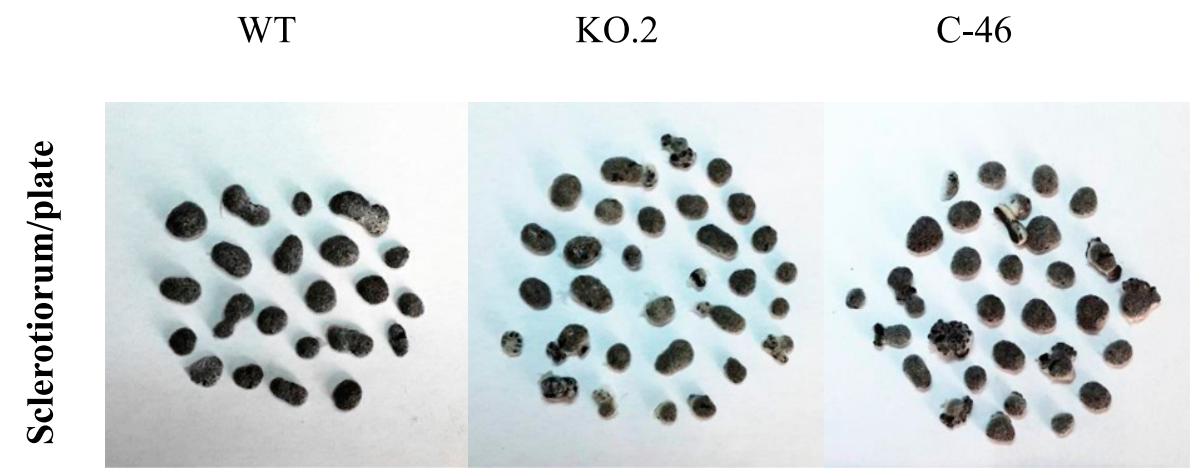

Fig. 3. Sclerotial morphology of the wild-type, Ss-FoxE2 knock-out mutant KO.2 and Ss-FoxE2 complemented mutant C-46.

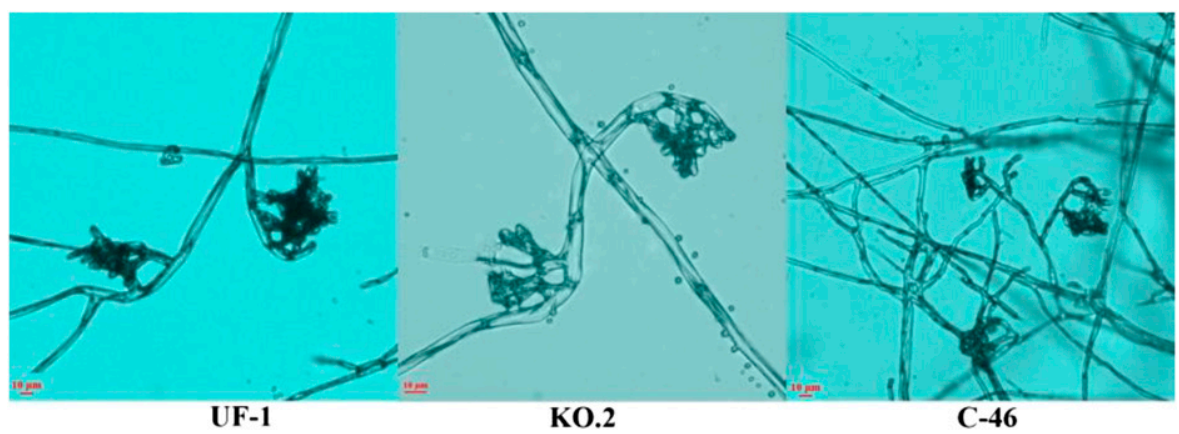

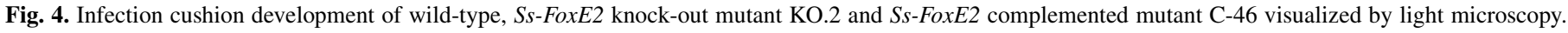
The scale bar is $10 \mu \mathrm{m}$. 
strains) were produced on autoclaved smashed potatoes with $1.5 \%$ agar at room temperature. These sclerotia were used to assess carpogenic germination. To induce apothecia, approximately 60 dry sclerotia were uniformly spread in a glass petri dish $(15 \mathrm{~cm} \emptyset)$ containing autoclaved water-saturated vermiculite. Plates were placed in a $15^{\circ} \mathrm{C}$ incubator with constant cool white fluorescent lights. After about 6 weeks, apothecial morphology was checked and the observation continued for 8 weeks.

Pathogenicity test. For pathogenicity analysis, 7-week-old tomato leaves were inoculated with an agar plug of wild type, Ss-FoxE2 knock-out and complementation strains and incubated at room temperature in petri dish with wet filter papers for 3 days to promote symptom development. In each experiment, each strain was used to inoculate at least three leaves from three plants and the pathogenicity assays were performed three times.

\section{RESULTS}

Identification of $\boldsymbol{S s - F o x E 2}$. Forkhead proteins play a key role in animal and fungal development, and in order to clarify the functions of FOX TFs in S. sclerotiorum, we conducted sequence analysis and functional characterization of the FoxE2 gene in S. sclerotiorum. Through PFAM analysis, four genes (SS1G_05834, SS1G_00580, SS1G_07360, and SS1G_10612) encoding proteins containing a forkhead domain (InterPro number: IPR001766 and PFAM number: PF00250) and six genes (SS1G_11744, SS1G_10786, SS1G_04069, SS1G_02656, SS1G_01850, and SS1G_00521) encoding proteins containing a forkhead associated domain (InterPro number: IPR000253 and PFAM number: PF00498) were identified in the $S$. sclerotiorum genome, among them, SS1G_10612 including both a forkhead domain and a forkhead associated domain. We chose to characterize the functions of two forkhead domain genes, SS1G_00580 (Ss-FoxE1; the focus of an independent study) and SS1G_05834 (Ss-FoxE2), because these two genes are specific orthologs of $M o F K H 1$ and $M o H C M 1$ which have been reported to be important in $M$. oryzae development. So, we speculated that these two genes may also have important functions in S. sclerotiorum. SS1G_05834 (Ss-FoxE2) encodes 450 amino acids containing a forkhead domain (amino acids 213 to 287). Sequence alignments with proteins previously described as forkhead box proteins in Mus musculus (FoxN3, NP_899009.2), Homo sapiens (FoxK1, NP_001032242.1), Danio rerio (FoxI1, NP_944600.1), and Acremonium chrysogenum (FKH1, AAP35674.1) (Katoh 2004; Samaan et al. 2010; Schmitt et al. 2004; Solomon et al. 2003) confirmed that Ss-FoxE2 had the conserved "NSIRHNLS" motif of the forkhead domain.

Deletion of the Ss-FoxE2. The experimental strategy to replace the Ss-FoxE2 locus with the $h p h$ gene was as described in the Materials and Methods. The gene replacement construct was used to transform wild-type $S$. sclerotiorum protoplasts. A total of 15 hygromycin-resistant transformants were obtained and further purified by three rounds of hyphal-tip transfer. Homologous recombinants were verified by PCR screening and Southern blot analysis (Fig. 1). For PCR screening, four sequence fragments were used for verification. The first fragment is a 712-bp sequence derived from $S s-F o x E 2$, the second is a 650-bp sequence derived from the $h p h$ gene, the third a 1,200-bp left flank and partial $h p h$ sequence, and the fourth a 1,300-bp right flank and partial hph sequence. Transformants lacking an amplicon from the Ss-FoxE2 primers and producing amplicons from the remaining primer pairs were used for further Southern blot analysis. The results showed that the expected fragment hybridized using probe 1 in the wild type but failed to hybridize with any fragments in the two putative genereplacement strains (KO.2 and KO.5; Fig. 1). In order to confirm that elimination of the corresponding Ss-FoxE2 sequences from the genome led to the lack of hybridization with probe 1 , a second probe (probe 2) was used for hybridization (Fig. 1). Probe 2 hybridized with the expected band in the Ss-FoxE2 delete strain KO.2 and KO.5 but not in the wild type. This result is consistent with the PCR screening data.

Transformant KO.2 was selected as a representative $S s$-FoxE2 gene-replacement strain and was designated as a recipient for complementation via transformation with $\mathrm{pD}-\mathrm{NEO} 1$ vector. The pD-NEO1 vector contains the integral genomic Ss-FoxE2 gene and a geneticin resistance marker. Probes 1 and 2 were also used for complementation strain screening (Fig. 1).

Ss-FoxE2 is dispensable for mycelial growth and sclerotium formation. No obvious differences were detected in mycelial growth on PDA medium between the wild type and Ss-FoxE2 mutant KO.2 (Fig. 2). Additionally, deletion of the Ss-FoxE2 coding sequence from $S$. sclerotiorum did not affect the development of mature sclerotia growing in PDA culture (Fig. 3). The number and biomass of sclerotia from the Ss-FoxE2 mutant are not reduced on PDA compared with the wild type (data not shown). Ss-FoxE2 deletion did not have a noticeable effect on the hyphae morphology and infection cushion development (Figs. 2, 3, and 4). These results suggest that Ss-FoxE2 is unnecessary for vegetative growth and development in $S$. sclerotiorum.

Ss-FoxE2 knockout mutant has no obvious change in virulence. To determine whether $S s$-FoxE2 regulates pathogenesis of $S$. sclerotiorum, we inoculated tomato leaves with the wild type, $\triangle S$ s-FoxE2 mutant KO.2 and complementation strain. All three

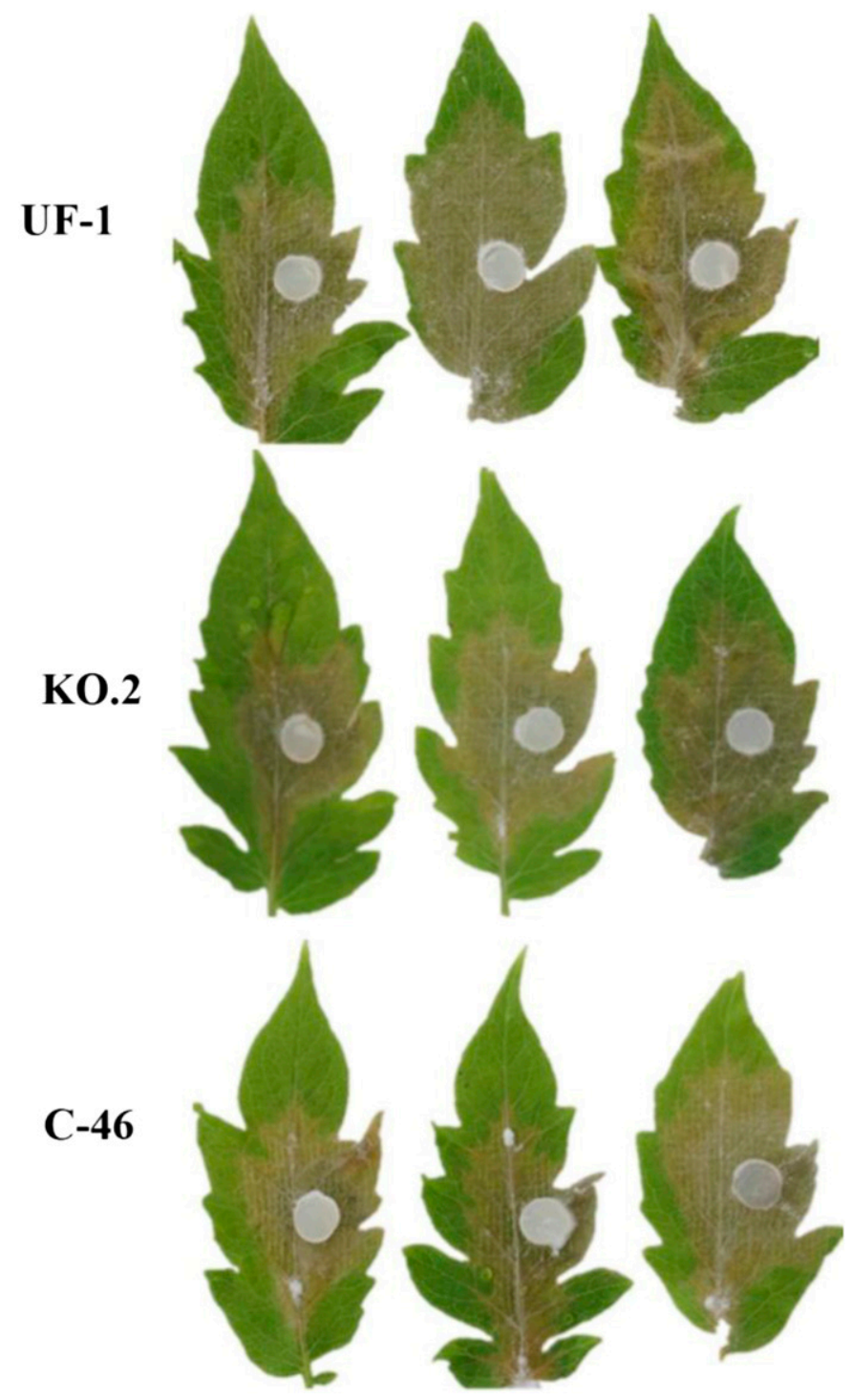

Fig. 5. Pathogenicity analysis. UF-1: wild-type strain; KO.2: Ss-FoxE2 knockout mutant; and C-46: Ss-FoxE2 complemented mutant C-46. 
strains infected unwounded tomato leaves and caused expanding, necrotic lesions within 3 days after inoculation. Under the same conditions, typical infection lesions were observed on all tomato leaves, and there was no obvious difference among the lesions caused by wild type, KO.2, and complementation strain (Fig. 5). These results indicate that $S s$-FoxE2 does not affect the infection or colonization of host tissue.

The $S s-F o x E 2$ gene is necessary for apothecia formation. The deletion of Ss-FoxE2 has no effect on vegetative reproduction and virulence in $S$. sclerotiorum, but a significant difference was observed on apothecia formation between the wild type and $\triangle S$ s-FoxE2 mutant (Fig. 6). Under the conditions of our assays, the sclerotia of wild type, $\triangle S s$-FoxE2 mutant, and complemented strain were used to assess carpogenic germination of sclerotia. Following 6 weeks of incubation, mature sclerotia of wild type germinated and produced fertile apothecia. No apothecia were observed on $\triangle S s$-FoxE2 mutant sclerotia, while apothecia were recovered from the complementation strain and the size of apothecia is smaller than the wild type but there was no difference on quantity. The phenotypes of a second independent knock-out mutant and three additional complementation strains were consistent (data not shown).

Expression dynamics of the Ss-FoxE2 gene. The Ss-FoxE2 knock-out mutant did not generate apothecia. This suggests that
Ss-FoxE2 may be involved in the formation of apothecia during the life cycle of $S$. sclerotiorum. We evaluated the expression levels of Ss-FoxE2 in various developmental stages of S. sclerotiorum. Results showed that the expression levels of $S s-F o x E 2$ in apothecia tissues were much higher than that in other developmental stages (Fig. 7). The relative expression levels of Ss-FoxE2 in three apothecia stages relative to vegetative hyphae were $49.88,79.82$, and 90.48 , respectively. The highest relative expression level among the sampled sclerotia stages was only 7.37. This result indicated that Ss-FoxE2 was expressed significantly higher in apothecia stages than that in other life stages.

\section{DISCUSSION}

Ss-FoxE2 encodes a FOX TF with orthologs in other fungi involved in regulating development, pathogenicity, and stress response (Lee et al. 2005; Park et al. 2010, 2014). FOX proteins are not the largest family of TFs; however, they play a prominent function in a number of biological processes (Carlsson and Mahlapuu 2002). Although well-annotated FOX TFs have been characterized in other fungi (Ostrow et al. 2014; Park et al. 2014), the functions of FOX TFs still remain unknown in S. sclerotiorum. Therefore, in this study we focused on determining the functions of the FOX TF gene in S. sclerotiorum
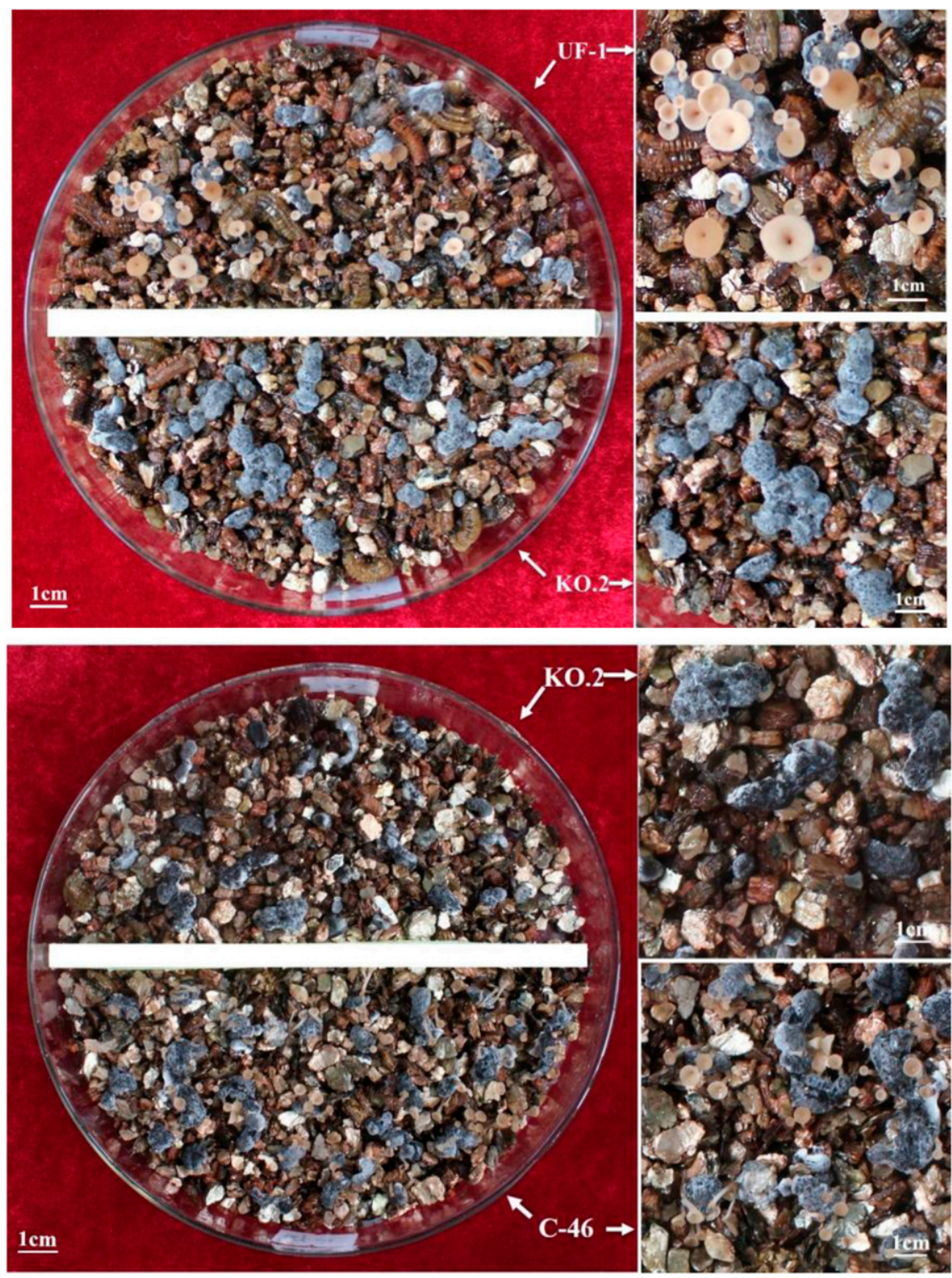

Fig. 6. Apothecia phenotypes of the wild type, Ss-FoxE2 knock-out mutant KO.2 and Ss-FoxE2 complemented mutant C-46. 
relative to asexual growth and development, sexual reproduction, and pathogenicity.

Previous studies have shown that FOX TFs play important roles in controlling cell cycle and morphogenesis (Park et al. 2014; Wang et al. 2015). Four putative FOX TF genes were found in Magnaporthe oryzae (MoFKH1, MoHCM1, MoFOX1, and MoFOX2). MoFKH1 and $M o H C M 1$ are significant in $M$. oryzae development, and MoFKH1 may further involvement in pathogenicity and stress response regulating in $M$. oryzae (Park et al. 2014). For many years, A. nidulans has been used as a model organism for studying growth and development of eukaryotic system, deletion of $f k h E$, a member of the forkhead family, resulted in impaired A. nidulans conidiophore formation in solid medium (Park et al. 2010). We designed the study to test our hypothesis that the Ss-FoxE2 gene plays an important role in the sexual reproduction in S. sclerotiorum. This hypothesis is supported based on the identification of $S s-F o x E 2$ gene as a developmentally regulated TF and the phenotypic characterization of Ss-FoxE2 gene knock-out mutants showing that Ss-FoxE2 is necessary for apothecia formation.

In $S$. cerevisiae, the $f k h 1$ and $f k h 2$ mutants show a constitutive pseudohyphal morphology, suggesting that Fkh1 and Fkh2 may help switch growth forms (Zhu et al. 2000). MoFKH1 in M. oryzae (Park et al. 2014), Fkh2 in S. pombe (Bulmer et al. 2004), and $\mathrm{CaFKH} 2$ in Candia albicans (Bensen et al. 2002) are homologs of Fkh1 and Fkh2 from S. cerevisiae. Deletion of MoFKH1 resulted in reduced mycelial growth and conidial germination, abnormal septation and stress response, and reduced virulence in $M$. oryzae (Park et al. 2014). Fkh2 in S. pombe is required for normal cell growth and division, and Fkh2 in $S$. cerevisiae is necessary for the morphogenesis of true hyphal as well as yeast cells (Bensen et al. 2002; Bulmer et al. 2004). In S. sclerotiorum, we chose to focus on Ss-FoxE2 as it is orthologous to Fkhl in M. oryzae and Fkh2 in $S$. cerevisiae. The lack of apothecium development in the $s s-f o x E 2$ loss of function is consistent with a role in morphogenesis and possibly cell cycle regulation.

In S. sclerotiorum life cycle, ascospores germinate to produce hyphae first, and then a large number of hypha aggregate to form a sclerotium. The sclerotium serves as supporting medium, and when environmental conditions are suitable apothecia will be formed and ascospores will be forcibly discharged to begin the next life cycle. In this study, sclerotia can be formed in the $\triangle S s$-FoxE2 mutant. In 6 weeks, mature sclerotia of the wild type germinated and produced fertile apothecia. No apothecia appeared on $\triangle S s$-FoxE2 mutant sclerotia, while apothecia were recovered from the complementation strain. Other genes influencing sclerotium development or

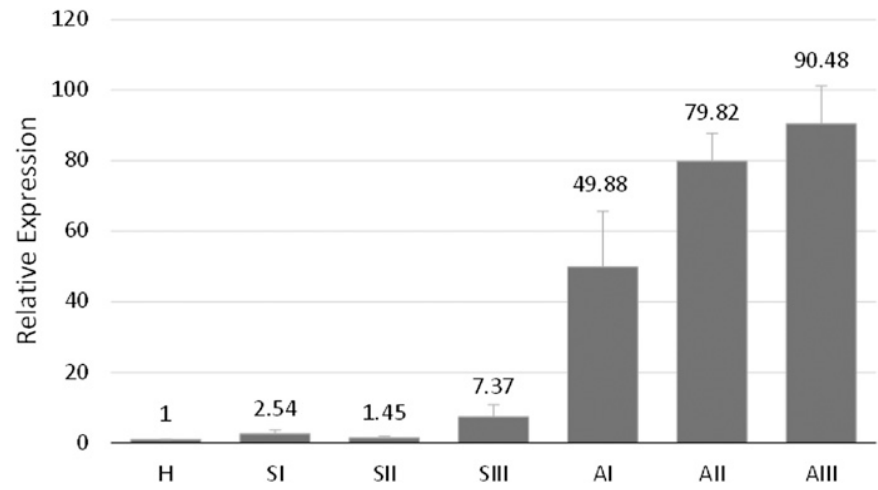

Fig. 7. Real-time reverse transcription-polymerase chain reaction (RT-PCR) analysis of Ss-FoxE2 transcript in different developmental stages of Sclerotinia sclerotiorum $. \mathrm{H}=$ hyphae; $\mathrm{SI}=$ sclerotia stages $\mathrm{S} 1$ to $\mathrm{S} 2$; $\mathrm{SII}=$ sclerotia stages $\mathrm{S} 3$ to $\mathrm{S} 4 ; \mathrm{SIII}=$ sclerotia stages $\mathrm{S} 5$ to $\mathrm{S} 6$; $\mathrm{AI}=$ apothecium stages $\mathrm{A} 1$ to $\mathrm{A} 3$; $\mathrm{AII}=$ apothecium stages $\mathrm{A} 4$ to A5; and AIII = apothecium stages A6 to A7. The expression level of Ss-FoxE2 cDNA measured by real-time RT-PCR was normalized to that of $\mathrm{Ss}$-Histone-H3 cDNA from each developmental stage. Hyphae RNA served as the reference sample and was assigned a value of 1 . Bars indicate standard error. function have been identified in S. sclerotiorum and Botrytis cinerea, including nicotinamide adenine dinucleotide (NADPH) oxidases (SsNox1 and SsNox2) and BcNoxA and BcNoxB (Kim et al. 2011; Segmüller et al. 2008). In this research, phenotypic characterizations of the loss-of-function, $\triangle S s$-FoxE2 mutant is consistent with that of a positive regulator of apothecia formation of sexual development. The data of $S s-F o x E 2$ expression showed that the expression levels of $S s-F o x E 2$ in apothecia tissues were much higher than that in other developmental stages, while the relative expression levels of Ss-FoxE2 in three apothecia stages were more than 10 times higher than that in sclerotia stage. Examining asexual development, the dry weight of sclerotia developed from the Ss-FoxE2 knock-out strain was similar to the wild type, but there was a small nonstatistical difference (Supplementary Table S1; Supplementary Fig. S1). The average weight of KO.2 was $0.26 \mathrm{~g}$ per plate and the wild type was $0.28 \mathrm{~g}$, and the number of sclerotia was consistent with the weight; the wild type averaged 28 per plate, and the KO. 2 strain, 26 per plate. Therefore, we conclude that the Ss-FoxE2 plays no, or a small, role in sclerotium development but an important role in apothecia development, especially in carpogenic germination of $S$. sclerotiorum.

In conclusion, the $S s$-FoxE2 gene in $S$. sclerotiorum was characterized as a FOX TF required for apothecia development. The results in this study provided evidence that $S$-FoxE2 played a critical role in sexual reproduction. This is consistent with other fungal FoxE orthologs. Further studies will focus on understanding upstream signaling pathways and the function of downstream genes, which will help to understand the common and unique pathways adopted for variations in sexual developments in filamentous fungi and perhaps a means for interrupting these cycles for disease management.

\section{ACKNOWLEDGMENTS}

This work was financially supported by the National Natural Science Foundation of China (31271991, 31471730), the Ministry of Education Fund for the Doctoral of China (20120061110082), and the Special Program of the Ministry of Agriculture for Public Profession (201103016).

\section{LITERATURE CITED}

Adams, P. B., and Ayers, W. A. 1979. Sclerotinia sclerotiorum: Ecology of Sclerotinia species. Phytopathology 69:896-899.

Bensen, E. S., Filler, S. G., and Berman, J. 2002. A forkhead transcription factor is important for true hyphal as well as yeast morphogenesis in Candida albicans. Eukaryot. Cell 1:787-798.

Boland, G. J., and Hall, R. 1994. Index of plant hosts of Sclerotinia sclerotiorum. Can. J. Plant Pathol. 16:93-108.

Bulmer, R., Pic-Taylor, A., Whitehall, S. K., Martin, K. A., Millar, J. B. A., Quinn, J., and Morgan, B. A. 2004. The forkhead transcription factor Fkh2 regulates the cell division cycle of Schizosaccharomyces pombe. Eukaryot. Cell 3:944-954.

Carlsson, P., and Mahlapuu, M. 2002. Forkhead transcription factors: Key players in development and metabolism. Dev. Biol. 250:1-23.

Chen, C., and Dickman, M. B. 2005. CAMP blocks MAPK activation and sclerotial development via Rap-1 in a PKA-independent manner in Sclerotinia sclerotiorum. Mol. Microbiol. 55:299-311.

Chen, C., Harel, A., Gorovoits, R., Yarden, O., and Dickman, M. B. 2004. MAPK regulation of sclerotial development in Sclerotinia sclerotiorum is linked with $\mathrm{pH}$ and cAMP sensing. Mol. Plant-Microbe Interact. 17:404-413.

Chet, I., and Henis, Y. 1975. Sclerotial morphogenesis in fungi. Annu. Rev. Phytopathol. 13:169-192.

Erental, A., Harel, A., and Yarden, O. 2007. Type 2A phosphoprotein phosphatase is required for asexual development and pathogenesis of Sclerotinia sclerotiorum. Mol. Plant-Microbe Interact. 20:944-954.

Georgiou, C. D., Patsoukis, N., Papapostolou, I., and Zervoudakis, G. 2006. Sclerotial metamorphosis in filamentous fungi is induced by oxidative stress. Integr. Comp. Biol. 46:691-712.

Harel, A., Bercovich, S., and Yarden, O. 2006. Calcineurin is required for sclerotial development and pathogenicity of Sclerotinia sclerotiorum in an oxalic acid-independent manner. Mol. Plant-Microbe Interact. 19:682-693.

Jurick, W. M., Dickman, M. B., and Rollins, J. A. 2004. Characterization and functional analysis of a cAMP-dependent protein kinase A catalytic subunit gene (pka1) in Sclerotinia sclerotiorum. Physiol. Mol. Plant Pathol. 64: 155-163. 
Kabbage, M., Yarden, O., and Dickman, M. B. 2015. Pathogenic attributes of Sclerotinia sclerotiorum: Switching from a biotrophic to necrotrophic lifestyle. Plant Sci. 233:53-60.

Katoh, M. 2004. Identification and characterization of human FOXK1 gene in silico. Int. J. Mol. Med. 14:127-132.

Kim, H. J., Chen, C. B., Kabbage, M., and Dickman, M. B. 2011. Identification and Characterization of Sclerotinia sclerotiorum NADPH Oxidases. Appl. Environ. Microbiol. 77:7721-7729.

Koranda, M., Schleiffer, A., Endler, L., and Ammerer, G. 2000. Forkhead-like transcription factors recruit Ndd1 to the chromatin of G2/M-specific promoters. Nature 406:94-98.

Kumar, R., Reynolds, D. M., Shevchenko, A., Shevchenko, A., Goldstone, S. D., and Dalton, S. 2000. Forkhead transcription factors, Fkh1p and Fkh2p, collaborate with Mcm1p to control transcription required for M-phase. Curr. Biol. 10:896-906.

Lam, E. W. F., Brosens, J. J., Gomes, A. R., and Koo, C. Y. 2013. Forkhead box proteins: tuning forks for transcriptional harmony. Nat. Rev. Cancer 13: 482-495.

Lee, B. Y., Han, S. Y., Choi, H. G., Kim, J. H., Han, K.-H., and Han, D. M. 2005. Screening of growth- or development-related genes by using genomic library with inducible promoter in Aspergillus nidulans. J. Microbiol. 43: 523-528.

Li, M., and Rollins, J. A. 2009. The development-specific protein (Ssp1) from Sclerotinia sclerotiorum is encoded by a novel gene expressed exclusively in sclerotium tissues. Mycologia 101:34-43.

Li, M., and Rollins, J. A. 2010. The development-specific ssp1 and ssp2 genes of Sclerotinia sclerotiorum encode lectins with distinct yet compensatory regulation. Fungal Genet. Biol. 47:531-538.

Ostrow, A. Z., Nellimoottil, T., Knott, S. R., Fox, C. A., Tavare, S., and Aparicio, O. M. 2014. Fkh1 and Fkh2 bind multiple chromosomal elements in the $S$. cerevisiae genome with distinct specificities and cell cycle dynamics. PLoS One 9:e87647.

Park, J., Kong, S., Kim, S., Kang, S., and Lee, Y. H. 2014. Roles of forkheadbox transcription factors in controlling development, pathogenicity, and stress response in Magnaporthe oryzae. Plant Pathol. J. 30:136-150.

Park, M. H., Kim, H. Y., Kim, J. H., and Han, K. H. 2010. Gene structure and function of $f k h E$ a forkhead gene in a filamentous fungus Aspergillus nidulans. Korean J. Mycol. 38:160-166.

Patsoukis, N., and Georgiou, C. D. 2007. Effect of sulfite-hydrosulfite and nitrite on thiol redox state, oxidative stress and sclerotial differentiation of filamentous phytopathogenic fungi. Pestic. Biochem. Physiol. 88:226-235.

Pramila, T., Wu, W., Miles, S., Noble, W. S., and Breeden, L. L. 2006. The Forkhead transcription factor Hcm 1 regulates chromosome segregation genes and fills the S-phase gap in the transcriptional circuitry of the cell cycle. Genes Dev. 20:2266-2278.
Proctor, R. H., Hohn, T. M., and McCormick, S. P. 1997. Restoration of wildtype virulence to Tri5 disruption mutants of Gibberella zeae via gene reversion and mutant complementation. Microbiology 143:2583-2591.

Rollins, J. A. 2003. The Sclerotinia sclerotiorum pac1 gene is required for sclerotial development and virulence. Mol. Plant-Microbe Interact. 16: 785-795.

Samaan, G., Yugo, D., Rajagopalan, S., Wall, J., Donnell, R., Goldowitz, D., Gopalakrishnan, R., and Venkatachalam, S. 2010. Foxn3 is essential for craniofacial development in mice and a putative candidate involved in human congenital craniofacial defects. Biochem. Biophys. Res. Commun. 400:60-65.

Sambrook, J., and Russell, D. W. 2001. Molecular Cloning. A Laboratory Manual. 3rd ed. Cold Spring Harbor Laboratory Press, Cold Spring Harbor, NY.

Schmitt, E. K., Hoff, B., and Kück, U. 2004. AcFKH1, a novel member of the forkhead family, associates with RFX transcription factor CPCR1 in the cephalosporin C-producing fungus Acremonium chrysogenum. Gene 342: 269-281.

Schmittgen, T. D., Zakrajsek, B. A., Mills, A. G., Gorn, V., Singer, M. J., and Reed, M. W. 2000. Quantitative reverse transcription-polymerase chain reaction to study mRNA decay: Comparison of endpoint and real-time methods. Anal. Biochem. 285:194-204.

Segmüller, N., Kokkelink, L., Giesbert, S., Odinius, D., van Kan, J., and Tudzynski, P. 2008. NADPH oxidases are involved in differentiation and pathogenicity in Botrytis cinerea. Mol. Plant-Microbe Interact. 21:808-819.

Shimeld, S. M., Degnan, B., and Luke, G. N. 2010. Evolutionary genomics of the Fox genes: Origin of gene families and the ancestry of gene clusters. Genomics 95:256-260.

Solomon, K. S., Kudoh, T., and Fritz, A. 2003. Zebrafish foxi1 mediates otic placode formation and jaw development. Development 130:929-940.

Wang, J. J., Qiu, L., Cai, Q., Ying, S. H., and Feng, M. G. 2015. Transcriptional control of fungal cell cycle and cellular events by Fkh2, a forkhead transcription factor in an insect pathogen. Sci. Rep. 5:10108.

Willetts, H. J., and Bullock, S. 1992. Developmental biology of Sclerotinia. Mycol. Res. 96:801-816.

Willetts, H. J., and Wong, J. A. L. 1980. The biology of Sclerotinia sclerotiorum, $S$. trifoliorum, and $S$. minor with emphasis on species nomenclature. Bot. Rev. 46:101-165.

Xiao, X., Xie, J., Cheng, J., Li, G., Yi, X., Jiang, D., and Fu, Y. 2014. Novel secretory protein Ss-Caf1 of the plant-pathogenic fungus Sclerotinia sclerotiorum is required for host penetration and normal sclerotial development. Mol. Plant-Microbe Interact. 27:40-55

Zhu, G., Spellman, P. T., Volpe, T., Brown, P. O., Botstein, D., Davis, T. N., and Futcher, B. 2000. Two yeast forkhead genes regulate the cell cycle and pseudohyphal growth. Nature 406:90-94. 